\title{
Allier plurilinguisme et variation dans un $M O O C$ : enjeux et contraintes
}

\section{Combining plurilingualism and variation in a MOOC: issues and constraints}

\section{Combinando plurilingüismo y variación en un MOOC: desafíos y limitaciones}

https://doi.org/10.52358/mm.vi7.215

Carole Cattin, assistante suppléante

Université de Genève, Suisse

Carole.Cattin@unige.ch

RÉSUMÉ

Cet article, dont le but est de faire ressortir quelques enjeux pédagogiques dans le passage de deux cours universitaires à un $M O O C$, se base sur la réflexion entamée dans notre travail de mémoire qui a été réalisé dans le cadre d'un projet $M O O C$ intitulé «Langues et diversité : de la variation au plurilinguisme » actuellement conçu à l'Université de Genève. C'est donc en nous appuyant sur ce projet axé sur la diversité linguistique que nous allons mettre l'accent sur certaines adaptations à apporter, tant sur le plan des contenus que du discours, pour que les enseignements donnés en présentiel soient adaptés au dispositif d'un MOOC.

Mots-clés : $M O O C$, diversité linguistique, variation, plurilinguisme

\section{ABSTRACT}

This article highlights some pedagogical issues that arise in the transition from two university courses to a MOOC. This reflection started with our master thesis on a MOOC project focussing on linguistic diversity entitled "Languages and diversity: from variation to plurilingualism," which is currently in progress at the University of Geneva. Based on this project, we suggest reflecting on certain adjustments in content and discourse for in-person classes to be adapted to a MOOC.

Keywords: MOOC, linguistic diversity, variation, plurilingualism 
RESUMEN

En este artículo, intentaremos poner de relieve algunos retos educativos que surgen de manera obligatoria en la transición de dos cursos universitarios a un MOOC. Este trabajo, empezado en nuestra tesis de máster, se lleva a cabo en el marco de un proyecto MOOC cuyo título es "Idiomas y diversidad: de la variación al plurilingüismo", que se realiza actualmente en la Universidad de Ginebra. Basándonos en este proyecto centrado en la diversidad lingüística, proponemos una reflexión necesaria sobre las adaptaciones, tanto en términos de contenido como en términos de tipo de habla, para que las lecciones impartidas en persona se adapten al sistema MOOC.

Palabras clave: MOOC, diversidad lingüística, variación, plurilingüismo

\section{Introduction'}

Cette contribution a pour objectif de présenter la réflexion menée dans le cadre du passage de deux cours universitaires - l'un traitant du plurilinguisme et l'autre de la variation en français oral - à un $M O O C$ ayant à la fois pour intention de traiter de ces deux thématiques de manière miroir sur le plan du contenu, mais aussi de faire appel à la diversité linguistique dans les interventions qui le constituent. Concrètement, nous nous proposons de présenter quelques enjeux pédagogiques du passage de ces deux enseignements à un $M O O C$ en mettant notamment en avant certains défis qu'une telle adaptation entraine. Cette réflexion, entamée dans notre travail de mémoire, s'insère dans le cadre d'un projet $M O O C$ intitulé « Langues et diversité : de la variation au plurilinguisme », projet retenu à la suite d'un appel lancé par le Rectorat de I'Université de Genève à la fin 2019.

Afin de répondre à notre question de recherche qui consiste à s'interroger sur les enjeux et les contraintes de conception pédagogique d'un $M O O C$ offert dans une diversité de langues et variétés, la présente contribution s'articulera en deux parties. La première sera consacrée à la présentation du projet de MOOC "Langues et diversité : de la variation au plurilinguisme ". Nous présenterons tout d'abord la thématique centrale de ce cours avant d'en décrire la structure. La deuxième partie sera quant à elle consacrée aux enjeux et contraintes du passage des deux cours universitaires traitant de la diversité à ce MOOC. Nous verrons dans un premier temps que ce passage demande des adaptations tant sur le plan du contenu que du discours, puis nous nous intéresserons à la façon dont la diversité linguistique sera intégrée dans ce MOOC. À noter que si des questions de scénarisation interviennent bel et bien dans cette contribution, celle-ci se focalise davantage sur la structuration et l'adaptation du contenu pour une offre à distance.

\footnotetext{
${ }^{1}$ Nous tenons à remercier l'équipe enseignante du MOOC "Langues et diversité : de la variation au plurilinguisme » - à savoir le professeur Laurent Gajo, la professeure Isabelle Racine et la docteure Mariana Fonseca - de nous avoir permis de rejoindre le projet. Nous remercions également la Cellule MOOC de l'Université de Genève pour son aide dans la conception du cours. Enfin, notre gratitude va aux éditeurs et éditrices de ce volume ainsi qu'aux deux relecteurs et relectrices anonymes, et à Isabelle Racine et Mariana Fonseca pour leurs précieux commentaires qui nous ont permis d'améliorer notre contribution.
} 


\section{Le projet de $M O O C$ « Langues et diversité : de la variation au plurilinguisme »}

L'originalité de ce $M O O C$, dont la conception est en cours à l'Université de Genève, réside dans sa volonté d'allier plurilinguisme (diversité entre les langues) et variation (diversité à l'intérieur d'une langue, en l'occurrence le français) en présentant ces notions comme faisant partie d'un continuum, tel que cela a été envisagé dans un ouvrage récemment publié et intitulé Variation, plurilinguisme et évaluation en français langue étrangère (Gajo et al. 2019). Cette continuité - jusqu'à présent peu documentée dans la littérature scientifique - est notamment traitée par Coste (2019) dans l'introduction de cet ouvrage. Selon cet auteur, les normes ne peuvent qu'exister parce que les langues varient, tant sur le plan intralinguistique (variation autour d'une même langue) qu'interlinguistique (variation entre différentes langues). II ajoute que c'est cette variation qui nous pousse à nous interroger sur la notion même de langue, puisque

«l'insistance est plus sur la pluralité interne des "langues", sur les continuums avec d'autres
"langues" et sur l'existence pour les usagers de ressources langagières hétérogènes, certes non
pas indistinctes, mais qui ne sauraient être strictement cataloguées en termes de langues
homogènes, clairement délimitées et normées »(Coste 2019, p. 20-21).

Afin d'illustrer cette continuité entre les deux thématiques que sont le plurilinguisme et la variation, le projet d'un $M O O C$ - qui s'appuie principalement sur deux enseignements de Bachelor de français langue étrangère normalement dispensés en présentiel à l'Université de Genève (un enseignement sur le plurilinguisme et l'autre sur la variation) - a été imaginé en six modules qui fonctionnent en miroir, puisque trois se situent sur l'axe du plurilinguisme et trois sur l'axe de la variation du français oral. Les mêmes aspects sont donc appréhendés une première fois sous l'angle du plurilinguisme et une seconde fois sous celui de la variation. La figure 1 schématise la structure générale de ce MOOC : 


\section{Figure 1}

Structure du MOOC «Langues et diversité : de la variation au plurilinguisme »

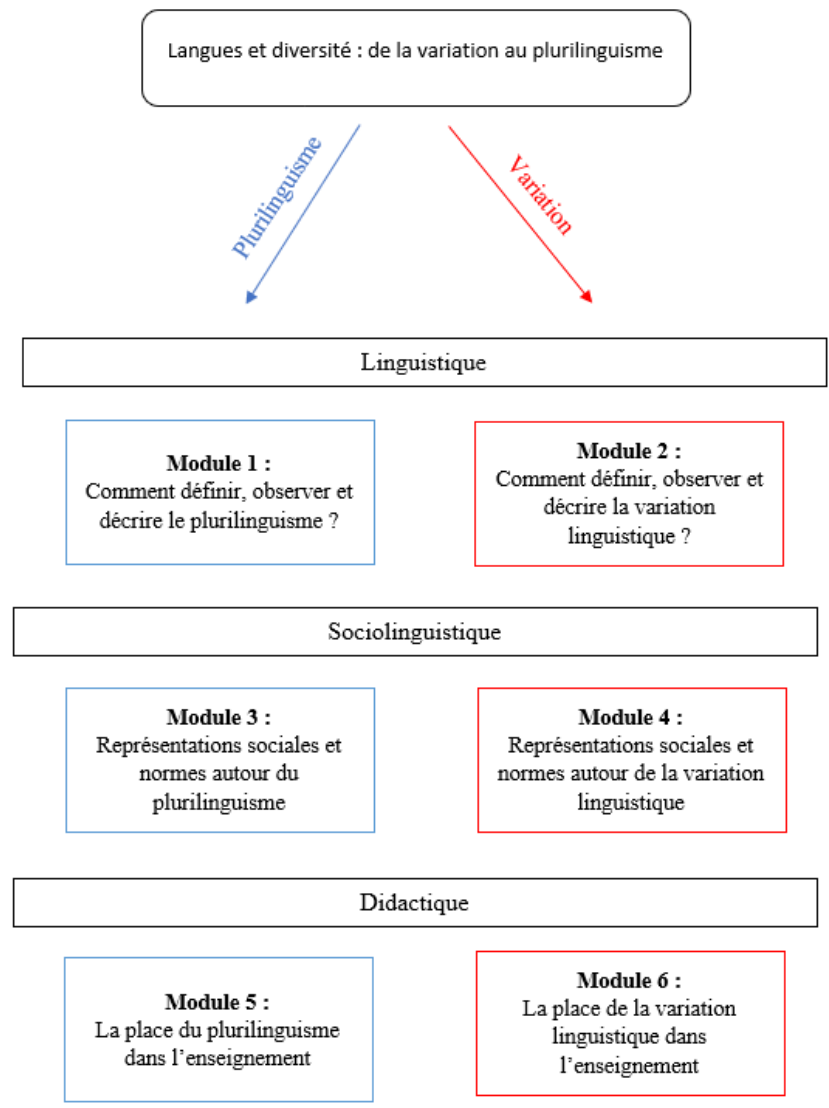

Note. Source : Cattin (2021)

Comme l'illustre la figure 1, la diversité linguistique est déclinée en trois axes : le premier est consacré à la description de cette diversité entre les langues (module 1) et à l'intérieur d'une même langue (module 2) alors que les deuxième et troisième axes permettent de mettre en avant les impacts de ces deux aspects de la diversité linguistique sur le plan sociétal (modules 3 et 4 ) puis sur le plan didactique (modules 5 et 6). Comme le $M O O C$ est intitulé «Langues et diversité : de la variation au plurilinguisme », il peut paraitre étonnant de prime abord que, pour chacun des trois axes, les modules portant sur le plurilinguisme précèdent ceux traitant de la variation. Ce choix est commenté par Daniel Coste dans un entretien de la leçon de bienvenue du MOOC. En effet, si les questionnements autour de la variation ne sont pas nouveaux, ils bénéficient en quelque sorte d'un second souffle amené par l'essor des travaux, plus récents, sur le plurilinguisme. La manière dont ces deux aspects de la diversité - inter et intra langue(s) - sont articulés est quant à elle explicitée plus spécifiquement dans les introductions et conclusions spécifiques à chacun des trois axes.

C'est dans le cadre de ce projet que notre travail de mémoire a été réalisé. Dans un premier temps, nous avons réfléchi sur les modifications à apporter, tant sur le plan des contenus que du discours, pour adapter un enseignement universitaire au dispositif MOOC. Nous avons ensuite tenté de mettre en pratique cette 
réflexion en développant un septième module, complémentaire aux six présentés ci-dessus. La réflexion sur les enjeux pédagogiques que nous proposons ici s'est nourrie de celle menée autour de la réalisation de ce module supplémentaire².

\section{Enjeux et contraintes du passage de deux cours universitaires traitant de la diversité linguistique à un MOOC}

Comme nous le verrons dans cette section, le passage de cours en présentiel à un $M O O C$ est complexe à plusieurs égards, à tel point qu'il serait peut-être plus adéquat de parler de "refonte " plutôt que de "passage ». En nous appuyant sur les deux enseignements universitaires sur lesquels se fonde principalement le MOOC "Langues et diversité : de la variation au plurilinguisme ", nous nous concentrerons dans un premier temps sur les différences de temporalité et de public se rapportant à ces deux types de cours. Par la suite, nous évoquerons quelques pistes permettant d'introduire du plurilinguisme et de la diversité dans un dispositif de MOOC. À noter que la réflexion présentée ici se focalise volontairement sur l'adaptation des activités d'enseignement, et ce, bien que d'autres adaptations soient également nécessaires, comme celles liées aux activités d'apprentissage proposées aux participantes et participants.

Lors de la conception d'un $M O O C$, un premier défi consiste à traiter de la matière normalement présentée en classe dans un format temporel qui s'avère être bien plus restreint. Dans le cadre des deux principaux enseignements constituant le $M O O C$ "Langues et diversité : de la variation au plurilinguisme ", chaque enseignante et enseignant a normalement à sa disposition une heure et demie de cours lors des douze semaines que contient le semestre d'automne à l'Université de Genève. En ce qui concerne le format temporel des $M O O C$, si Achard (2016) relève qu'ils ont généralement une durée allant de quatre à douze semaines, il est toutefois utile de préciser que, comme l'indique la page Internet de l'Université de Genève sur les $M O O C$, la plupart des MOOC proposés par cet établissement ont une durée maximale de six semaines $^{3}$. C'est par exemple le cas du MOOC qui nous intéresse tout particulièrement ici (cf. figure 1 dans laquelle un module équivaut à une semaine). Achard (2016) précise en outre qu'une semaine de cours d'un $M O O C$ contient des capsules vidéo d'une durée totale d'une à deux heures, chaque vidéo allant généralement de cinq à douze minutes. Des études telles que celle de Guo et al. (2014) ont cependant démontré que, pour que les participantes et participants restent engagés tout au long du visionnage, les capsules vidéo ne devraient idéalement pas aller au-delà de six minutes. Dans le cadre du projet de MOOC "Langues et diversité : de la variation au plurilinguisme ", le choix a été fait de proposer des vidéos allant souvent au-delà de six minutes, mais dans lesquelles les participantes et participants devraient normalement être stimulés par la polyphonie des voix (entretiens, extraits sonores, etc.) et la diversité des langues/variétés. À noter encore qu'au vu des différents aspects temporels mentionnés ci-dessus, le passage d'un cours à un $M O O C$ implique non seulement une synthétisation drastique des contenus, mais aussi, à notre sens, une modification nécessaire du discours. II semble en effet évident qu'une compression du temps va de pair avec une condensation du discours, ce qui implique par exemple une réduction des reformulations et des répétitions normalement très présentes dans un cours dispensé en

\footnotetext{
${ }^{2}$ Ce module, qui ne sera pas présenté ici, est expliqué en détail dans notre mémoire (Cattin, 2021).

${ }^{3}$ https://moocs.unige.ch/presentation/
} 
présentiel. Mettre en place des vidéos courtes et claires tout en minimisant au maximum les reformulations représente donc un premier défi pédagogique.

Un deuxième type d'adaptation du discours, également indispensable, est lié à des contraintes relevant du public cible d'un $M O O C$, celui-ci étant différent de celui qui prend part aux cours en présentiel. En effet, si les deux enseignements qui constituent le MOOC «Langues et diversité : de la variation au plurilinguisme " sont normalement proposés à des étudiantes de Bachelor de français langue étrangère, les modules du MOOC peuvent, eux, être suivis par des participants et participantes de tous horizons, déjà sensibilisés ou non à la diversité linguistique. De manière plus générale, les $M O O C$ étant ouverts à toutes et à tous, leur mise en place doit nécessairement s'accompagner d'un important travail de vulgarisation de la part des enseignants et des enseignantes, et ce, sur deux plans. Premièrement, l'usage de termes trop spécialisés doit être évité et s'ils s'avèrent tout de même nécessaires, ils doivent toujours être explicités. Cette contrainte renvoie cependant au premier défi mentionné ci-dessus concernant les enjeux temporels. Le recours à un discours métaphorique, qui pourrait constituer une piste d'explication possible de certains termes, devrait toutefois, à notre sens, être évité. Les métaphores étant connotées culturellement, elles peuvent en effet être difficiles à comprendre pour les participantes et participants issus d'autres univers linguistiques et culturels. Deuxièmement, le travail de vulgarisation doit également être mené sur le plan de la sélection des contenus traités dans les MOOC, puisque le processus de didactisation - permettant de passer de données scientifiques à du contenu enseignable - doit permettre à un public n'ayant potentiellement aucune connaissance du domaine traité de comprendre les contenus proposés. C'est la raison pour laquelle il a été décidé, dans le projet de $M O O C$ « Langues et diversité : de la variation au plurilinguisme ", d'intégrer du matériel issu d'émissions télévisées, de podcasts, d'articles de presse, etc. Cependant, pour que les contenus restent tout de même universitaires, une attention particulière a été portée à ce que les contenus proposés dans ce matériel externe présentent le point de vue de spécialistes des thématiques concernées.

Un troisième enjeu pédagogique à prendre en compte lors de la mise en place d'un $M O O C$ - et qui exige également une adaptation sensible du discours - se rapporte à la scénarisation des capsules vidéo. En effet, afin que les cours soient adaptés au format à distance, ils se doivent d'être non seulement plus courts mais également scénarisés. À ce sujet, Peltier et Campion (2018) notent, entre autres, l'importance des choix de tournage (lieux, cadrage, etc.) et de la façon de s'adresser aux participantes et participants. Plus spécifiquement, ces auteurs relèvent par exemple l'importance pour les enseignantes et les enseignants de maintenir une relation avec le public afin que celui-ci puisse apprendre de manière efficace. Cela a directement pu être expérimenté par les concepteurs et conceptrices du MOOC « Langues et diversité : de la variation au plurilinguisme » lors d'un atelier de scénarisation qui leur a été proposé4. Dans le cadre de cet atelier, ils et elles ont en effet été encouragés à s'adresser directement aux participantes et aux participants, et à les interpeller en respectant un fil narratif composé d'une accroche, de contenus systématiquement suivis de relances et d'une conclusion. Cette façon de procéder revient par exemple à penser, lors de la rédaction des scripts, à poser des questions à un public qui ne sera pas réellement présent au moment des tournages, ce qui ne se fait bien entendu pas de la même façon en présentiel.

Enfin, des enjeux dans le passage d'un cours à un $M O O C$ résident également dans la prise en compte de la diversité linguistique et culturelle des participants et participantes. En effet, comme le relèvent Fonseca et Gajo (2020), les MOOC étant massifs, ouverts et en ligne, ils sont susceptibles d'être suivis par des personnes d'univers linguistiques et culturels variés. Par conséquent, une attention particulière devrait être

\footnotetext{
${ }^{4}$ Atelier proposé par la Cellule $M O O C$ de l'Université de Genève à toutes les équipes qui conceptualisent des MOOC dans cet établissement. Atelier mis en place par la souris verte (https://lasourisverte.ch/).
} 
portée à l'hétérogénéité linguistique du public. Au premier abord, ceci semble toutefois difficile, car cette diversité n'est pas représentée dans les langues des cours proposés sur les plateformes de MOOC qui, comme l'indiquent Depover et al. (2017), proviennent majoritairement des États-Unis. Ainsi, bien que Shah (2015) observe que les MOOC offerts en 2015 l'étaient dans seize langues, il constate également que la grande majorité des cours étaient proposés en anglais $(75 \%)^{5}$. Comme l'expliquent Stratton et Grace (2016), ce manque de diversité linguistique réduit l'accessibilité aux MOOC pour de nombreuses personnes :

«Previous studies of MOOC users have shown that the demographic characteristics of users in developed and developing countries are not as diverse as the rhetoric of MOOCs would promise. One factor that can constrain the demographic profile of MOOC users is a lack of universal access to MOOCs. We examine one potential challenge to universal access to MOOCs, the linguistic diversity of course offerings, to show that the existing infrastructure of MOOCs may preclude access and consequently shape the demographic profile of users. In this way, our study of MOOC infrastructure is complementary to studies of MOOC use. We find significant differences in quantities and types of MOOCs available in English and non-English languages. This finding indicates that MOOCs do not yet provide a broad array of educational opportunities for people without adequate English-language proficiency and therefore, MOOCs may have limited potential for use in international development outside English-speaking populations » (Stratton et Grace 2016, p. 8).

Pour pallier ce problème d'accessibilité des personnes non anglophones aux $M O O C$, ces auteurs proposent deux solutions : traduire des $M O O C$ déjà existants ou en créer des nouveaux dans d'autres langues que l'anglais. Les concepteurs et conceptrices du MOOC « Langues et diversité : de la variation au plurilinguisme » - qui ont non seulement pour but de traiter de la diversité sur le plan du contenu, mais aussi de l'inclure tout au long du MOOC - ont opté pour un cours dont la langue d'enseignement est majoritairement le français, mais dans lequel d'autres variétés et langues apparaissent à travers les voix des différents intervenants et intervenantes. À noter que si les passages proposés dans d'autres langues seront, dans un premier temps, sous-titrés en français, l'objectif est par la suite de proposer un sous-titrage multilingue à l'ensemble du MOOC. Plusieurs difficultés sont toutefois inhérentes à cette volonté d'introduire de la diversité dans ce $M O O C$ en particulier et dans les $M O O C$ en général. Des contraintes liées à la plateforme, à l'établissement, au budget, etc. - peuvent par exemple empêcher les concepteurs et conceptrices de faire appel à autant d'intervenantes et intervenants qu'initialement souhaité, ou rendre la disponibilité des sous-titres ardue. Une autre difficulté concerne les lectures qui accompagnent les capsules vidéo. En effet, si les vidéos et activités peuvent être sous-titrées dans d'autres langues, il semble difficile - voire impossible - d'imaginer traduire un article scientifique, par exemple. Ainsi, les participantes et participants ne maitrisant pas (suffisamment) la langue du document écrit seraient pénalisés par une activité de lecture. Une solution pourrait alors être de proposer des lectures dans diverses langues. S'il est évidemment impossible de couvrir toutes les langues des participants et participantes, l'équipe enseignante pourrait toutefois sélectionner des lectures dans deux ou trois langues. Cette façon de procéder permettrait à davantage de personnes d'avoir accès aux savoirs.

\footnotetext{
${ }^{5}$ Contre $80 \%$ en 2014 (Shah, 2015). L'auteur explique cette légère baisse par le fait que des établissements non anglophones ont commencé à se lancer dans la conception de MOOC.
} 


\section{Conclusion}

En conclusion, la réflexion présentée dans cette contribution a été l'occasion de mettre en avant quelques contraintes et limites rencontrées par les concepteurs et conceptrices du MOOC « Langues et diversité : de la variation au plurilinguisme » dans le passage des cours en présentiel au format MOOC. Sur le plan des contenus, il s'est avéré que la première nécessité a été de condenser et de repenser fortement la matière enseignée afin que les capsules vidéo demeurent courtes et donc adaptées à l'enseignement à distance. Plusieurs aspects ont ensuite été relevés en ce qui concerne l'adaptation du discours. Tout d'abord, un travail de vulgarisation semble être essentiel afin que les contenus puissent être compris par le vaste public susceptible de s'inscrire au MOOC. À cela s'ajoute les éléments se rapportant à la scénarisation de ce type de cours, scénarisation qui amène notamment les enseignantes et enseignants à changer leur façon de s'adresser à un public qui, dans ce cas, n'est pas directement visible. Pour finir, nous avons vu que s'il est essentiel d'introduire de la diversité linguistique dans les MOOC afin que des participants et participantes de langues premières variées puissent y avoir accès, certaines contraintes rendent cette tâche complexe. Quoi qu'il en soit, nous espérons que cette contribution - ainsi que l'ensemble de la réflexion menée dans ce projet - intéressera d'autres (futurs) concepteurs et conceptrices de $M O O C$, particulièrement celles et ceux ayant pour intention de mettre en avant la diversité linguistique. II est en effet utile d'avoir connaissance de certaines limites - liées aux plateformes de MOOC ou autres - avant de se lancer dans la conception d'un MOOC.

Pour ce qu'il en est du projet de $M O O C$ « Langues et diversité : de la variation au plurilinguisme », s'il n'y a pas de doutes sur le fait que de nombreux défis attendent encore l'équipe enseignante, il sera intéressant de voir, à terme, ce qu'elle tirera de cette expérience. Nous verrons donc si, comme le rapporte Dillenbourg (2017) au sujet des enseignantes et enseignants de l'École polytechnique fédérale de Lausanne ayant conçu un $M O O C$, leur manière d'enseigner en sera changée. À terme, il se peut par exemple qu'une partie du MOOC soit utilisée en classe inversée, enseignement hybride qui consiste, comme décrit par Bergmann et Sams (2012), à proposer aux étudiants et aux étudiantes de prendre connaissance de certains contenus à la maison avant de les approfondir en classe. 


\section{Liste de références}

Achard, P. (2016). Les MOOCs : cours en ligne et transformations des universités. Montréal : Presses de l'Université de Montréal.

Bergmann, J. et Sams, A. (2012). Flip Your Classroom: Reach Every Student in Every Class Every Day. États-Unis : ISTE; ASCD.

Cattin, C. (2021). Quelles adaptations pour le passage d'un cours universitaire « classique » à un MOOC? Étude en marge du projet MOOC "Langues et diversité : de la variation au plurilinguisme » mené à l'Université de Genève [Mémoire de maitrise, Université de Genève]. https://archive-ouverte.unige.ch/unige:151675

Coste, D. (2019). Le plurilinguisme entre variation et évaluation. Dans L. Gajo, J.-M. Luscher, I. Racine et F. Zay (dir.), Variation, plurilinguisme et évaluation en français langue étrangère (15-24). Berlin : Peter Lang.

Depover, C., Karsenti, T. et Komis, V. (2017). Pour comprendre les MOOCs : nature, enjeux et perspectives. Québec : Presse de l'Université du Québec.

Dillenbourg, P. (2017). Préface. Dans C. Depover, T. Karsenti et V. Komis (dir.). Pour comprendre les MOOCs : nature, enjeux et perspectives. Québec: Presse de l'Université du Québec.

Fonseca, M. et Gajo, L. (2020). Le plurilinguisme dans les MOOCs: profils d'usagers et fonctions du sous-titrage. Apprentissage des Langues et Systèmes d'Information et de Communication, 23(2). https://doi.org/10.4000/alsic.4816

Gajo, L., Luscher, J.-M., Racine, I. et Zay, F. (2019). Variation, plurilinguisme et évaluation en français langue étrangère. Berlin : Peter Lang.

Guo, P. J., Kim, J. et Rubin, R. (2014). How Video Production Affects Students Engagement: An Empirical Study of MOOC Videos. Dans M. Sahami, A. Fox, M. A. Hearst et M. T.H. Chi (dir.), Proceedings of the first ACM conference on Learning @ scale conference, 41-50. https://doi.org/10.1145/2556325.2566239

Peltier, C. et Campion, B. (2018). Constructions langagières, relation et cognition dans les capsules vidéo des MOOC : pour une revitalisation des possibilités éducatives de la vidéo. Distances et médiations des savoirs, 21, 1-21. https://doi.org/10.4000/dms.2125

Shah, D. (2015). MOOCs in 2015: Breaking Down the Numbers. EdSurge. https://www.edsurge.com/news/2015-12-28-moocs-in-2015-breaking-down-the-numbers

Stratton, C. et Grace, R. (2016). Exploring Linguistic Diversity of MOOCs: Implications for International Development. Proceedings of the Association for Information Science and Technology, 53(1), 1-10. https://doi.org/10.1002/pra2.2016.14505301071 